\title{
A study of the pain score following TRUS guided prostate biopsy with and without periprostatic lignocaine infiltration
}

\author{
Ali Q. ${ }^{1}$, Grower J. ${ }^{2}$ \\ ${ }^{1}$ Dr. Qutubuddin Ali, ${ }^{2}$ Dr. Jitendra Grower, both are Assistant Professor, Department of Surgery, L.N. Medical College, \\ Bhopal, MP, India.
}

Address for Correspondence: Dr. Qutubuddin Ali, Email: aliqutub74@icloud.com

\begin{abstract}
Introduction: Prostate cancer is one of the most common cancers in Western countries and is now increasing worldwide. Transrectal ultrasound guided prostate needle biopsy (TRUS) is the standard procedure to diagnose or exclude prostate cancer. TRUS-guided biopsy is associated with several complications and discomfort. We evaluated painless yet less invasive prostate biopsy. Material and Methods: A randomised study was conducted in 30 consecutive men divided into two groups. Group I consisted of fifteen patients who did not receive any analgesia, and another fifteen constituted study group, Group II, who received periprostatic infiltration of $1 \%$ lignocaine. Patient with suspected DRE findings or elevated PSA or both were advised to undergo TRUS guided prostate biopsy. Pain intensity during the procedure was evaluated using Visual Analogue Scale. A visual analog scale was used to assess the pain score. Statistical analysis of pain scores was performed using the Student $t$ test. Chi-square/ Fisher Exact test was used to find the significance of study parameters on categorical scale between two. Results: In Group 2, there was a marked reduction in the pain experienced during the procedure. The Chi-squared test for trend showed a significant association between the periprostatic infiltration of $1 \%$ lignocaine and reduction in pain on probe insertion and on taking the biopsy $(P=0.0001)$. Conclusion: The use of periprostatic infiltration of lignocaine before taking the needle biopsy significantly reduces the pain experienced by the patient during TRUS-guided prostate biopsy.
\end{abstract}

Keywords: Prostate, Transrectal ultrasound, Biopsy, Periprostatic infiltration.

\section{Introduction}

Prostate cancer $(\mathrm{PCa})$ is one of the most common cancers in men, with about 700,000 patients diagnosed worldwide each year [1]. Transrectal Ultrasound (TRUS) guided prostate biopsy is regarded as the gold standard for prostate cancer diagnosis as demonstrated by recent studies. The majority of patients perceive TRUS-guided prostate biopsy as a physically and psychologically traumatic experience [2].

The International Association for the Study of Pain has offered the following definition of pain: "Pain is an unpleasant sensory and emotional experience associated with actual or potential tissue damage, or described in terms of such damage" [3]. Two factors are usually responsible for pain during transrectal prostate biopsy:

Manuscript received: 10 $0^{\text {th }}$ April 2017

Reviewed: $18^{\text {th }}$ April 2017

Author Corrected: $24^{\text {th }}$ April 2017

Accepted for Publication: 30 ${ }^{\text {th }}$ April 2017 anal pain due to ultrasound probe, that causes pressure and stretching of muscle fibers, and pain at insertion of the needle through the prostate [3]. All of the painrating scales are reliable and valid. The well-known visual analogue scale (VAS) and numeric rating scale (NRS) for assessment of pain intensity agree well and are equally sensitive in assessing acute pain after surgery, and they are both superior to a fourpoint verbal categorical rating scale (VRS). The most commonly used anesthetic is lidocaine either in gel suspension or as an injectable preparation (periprostatic nerve block PPNB) [4] although there is no strong evidence to recommend the different types of anesthetics that may be used. Some authors doesn't use any types of anesthetics during transrectal prostate biopsy.

Men undergoing $\mathrm{PBx}$ experience considerable psychological stress, attributable to the fear of a 
potential cancer diagnosis, the anal route of penetration, that the subject organ is part of the sexual system, and the anticipated pain [5]. Therefore, many urologists have stressed the importance of anaesthesia as an integral part of $\mathrm{PBx}$ [6-8]. However, despite these considerations, using anaesthesia is still under debate because of doubt of its real benefits and the associated costs [9].

The present study was designed to compare the pain score following TRUS guided prostate biopsy with and without periprostatic lignocaine infiltration and future recommendation thereof.

\section{Material and Methods}

The present study was a randomised control study carried out in department of Surgery, L N Medical College and Hospital, Bhopal. A total of 30 consecutive men undergoing transrectal prostate biopsy in our department were enrolled for the study after having met the inclusion criteria and obtaining written informed consent. They were randomly divided into two groups Group I consisted of fifteen patients who did not receive any analgesia, and another fifteen constituted study group, Group II, who received periprostatic infiltration of $1 \%$ lignocaine.

Study design- Randomised control study.

Inclusion criteria- Patients who needed transrectal biopsy for suspected carcinoma of prostate either by digital rectal examination or elevated serum PSA level.

Exclusion criteria- Bleeding disorder, Acute prostatitis, Anal stenosis, Painful anal or perianal conditions

Collection of data- Male patients attending our outpatient department with history of lower urinary tract symptoms were evaluated and obtained written consent. A detailed clinical examination including digital rectal examination (DRE) was performed. Patient with suspected DRE findings or elevated PSA or both were advised to undergo TRUS guided prostate biopsy.
Before the procedure, routine hematological and biochemical investigations like hemoglobin, total differential leukocyte count, coagulogram, blood urea, serum creatinine, random blood sugar and urine culture and sensitivity were performed.

Methodology- Patients were examined in left lateral decubitus position. Digital rectal examination was performed before inserting the TRUS probe. In group 1 $(n=15)$ intrarectal KY jelly was applied, which has no analgesic action. In group II $(n=15)$, periprostatic infiltration of $1 \%$ lignocaine was performed. After transrectal placement of the probe, the prostate was imaged in the transverse and sagittal planes. With the prostate viewed in sagittal plane, an 8-inch 22 gauge Chiba needle was introduced under ultrasound guidance into the region of the bladder neck, at the base of prostate, just lateral to the junction between the prostate and seminal vesicle. Approximately $2.5 \mathrm{ml}$ of $1 \%$ lignocaine was injected at each site on each side of the prostate. The syringe was aspirated before injection to ensure that the vascular system has not been entered.

After injection, biopsy of the prostate was done with an 18-gauge biopsy needle fired by a spring action biopsy gun. Right after the procedure patient were asked to score pain caused by needle insertion into the prostate on a 10 point visual analogue scale (VAS), 0 being no pain and 10 being most severe pain ever experienced.

Statistical analysis- Descriptive statistical analysis was carried out in the present study. Student t test (two tailed, independent) was used to find the significance of study parameters on continuous scale between two groups Inter group analysis) on metric parameters, Chisquare/ Fisher Exact test was used to find the significance of study parameters on categorical scale between two. $\mathrm{P}$ value less than 0.05 was considered significant.

Statistical software- The statistical software SPSS 10.0 was used for the analysis of the data and Microsoft word and excel have been used to generate graphs, tables etc.

\section{Results}

In this prospective study a total of 30 patients, who presented with complaints of LUTS and with either elevated serum PSA or abnormal digital rectal examination were enrolled and subjected for prostate biopsy. The patients were divided into two groups. Group 1 received intrarectal K Y jelly \& Group 2 received peri-prostatic infiltration of $1 \%$ lignocaine before the procedure. The comparative data of the two groups of patients is shown in table 1 . 
Table-1: Comparative data of the two groups of patients.

\begin{tabular}{|c|c|c|c|}
\hline Variables & Group I & Group II & P value \\
& $(\mathbf{n = 1 5})$ & $\mathbf{1 5})$ & 0.750 \\
\hline Age in years & $68.20 \pm 8.21$ & $67.20 \pm 8.77$ & 0.469 \\
\hline Prostate Volume in cc & $42.70 \pm 16.76$ & $46.67 \pm 12.22$ & 0.247 \\
\hline Serum PSA in ng/ml & $43.68 \pm 62.53$ & $70.28 \pm 84.29$ & 0.001 \\
\hline VAS score & $5.53 \pm 2.75$ & $2.53 \pm 1.55$ & 0.117 \\
\hline Immediate complications & $66.7 \%$ & $26.7 \%$ & 0.483 \\
\hline
\end{tabular}

VAS - Visual Analogue Scale

Age distribution- The mean age of the patients in group 1 was 68.2 years \pm 8.21 , with a range of $51-85$. In group 2 range of age was 50 - 82 with mean age of 67.2 years \pm 8.77 . Both groups were comparable for their age $(\mathrm{p}=0.750)$. $(\mathrm{Table}-2$, Fig 1).

Table-2: Age distribution of patients.

\begin{tabular}{|c|c|c|c|c|c|c|}
\hline \multirow{2}{*}{ Age in years } & \multicolumn{2}{|c|}{ Group I } & \multicolumn{2}{|c|}{ Group II } & \multicolumn{2}{|c|}{ Total } \\
\hline & No & $\%$ & No & $\%$ & No & $\%$ \\
\hline $50-59$ & 2 & 13.3 & 4 & 26.7 & 6 & 20.0 \\
\hline $60-69$ & 5 & 33.3 & 4 & 26.7 & 9 & 30.0 \\
\hline $70-79$ & 7 & 46.7 & 6 & 40.0 & 13 & 43.3 \\
\hline $80 \&$ above & 1 & 6.7 & 1 & 6.7 & 2 & 6.7 \\
\hline Total & 15 & 100.0 & 15 & 100.0 & 30 & 100.0 \\
\hline
\end{tabular}

Pain score- The mean pain score in group I was $5.53 \pm 2.75$ whereas in group II mean value was $2.53 \pm 1.55$. Mean pain score was significantly less in group II when compared to group I ( $\mathrm{p}=.001)$. In study group $73.3 \%$ patient had mild pain during the biopsy whereas only $26.7 \%$ patients had mild pain, which was statistically significant.

Similarly one third of patients in control group had severe pain as compared to only $16.7 \%$ patients in study group who recorded severe pain. (Table-3).

Table-3: Comparison of pain score in two groups of patients.

\begin{tabular}{|c|c|c|c|c|c|c|}
\hline \multirow{2}{*}{ Pain score } & \multicolumn{2}{|c|}{ Group I } & \multicolumn{2}{|c|}{ Group II } & \multicolumn{2}{|c|}{ Total } \\
\hline & No & $\%$ & No & $\%$ & No & $\%$ \\
\hline None(0) & 0 & 0.0 & 0 & 0.0 & 0 & 0.0 \\
\hline Mild(1,2,3) & 4 & 26.7 & 11 & 73.3 & 15 & 50.0 \\
\hline Moderate $(4,5,6)$ & 6 & 40.0 & 4 & 26.7 & 10 & 33.3 \\
\hline Severe $(7,8,9,10)$ & 5 & 33.3 & 0 & 0.0 & 5 & 16.7 \\
\hline Total & 15 & 100.0 & 15 & 100.0 & 30 & 100.0 \\
\hline Mean $\pm \mathrm{SD}$ & \multicolumn{2}{|c|}{$5.53 \pm 2.75$} & \multicolumn{2}{|c|}{$2.53 \pm 1.55$} & \multicolumn{2}{|c|}{$4.03 \pm 2.67$} \\
\hline
\end{tabular}




\section{Discussion}

The prevalence of asymptomatic prostate cancer in the population increases with age. As much as $64 \%$ of men between the age of 60-70 years harbor cancerous cells in the prostate [10]. Like all cancers, prostate cancer is best managed when diagnosed early as both the recurrence-free survival and the cancer-specific survival are inversely related to the stage of disease at detection [11].

Many studies done so far are supporting the role of local anesthesia in reducing pain and complications following transrectal ultrasound guided prostate biopsy. This study is a prospective study done to establish the role of peri-prostatic infiltration of $1 \%$ lignocaine in reducing pain during the procedure \& reducing incidence of morbities and complications associated with TRUS guided biopsy. Our study support the hypothesis that peri-prostatic infiltration of $1 \%$ lignocaine reduces the pain. Bulbul et al [12] in their study on 72 patients, respectively, evaluated the effect of periprostatic local anesthetic injection on pain relief during prostate biopsy. Saad et al [13] observed that the rectal administration of lignocaine gel reduces the pain experienced during the biopsy. Desgrandchamps et al [14] disagree and state that the rectal administration of lignocaine gel has no benefit over placebo.

Wang $\mathrm{J}$ et al [15] and Woo et al [16] concluded that combined modalities show better analgesic efficacy than periprostatic nerve block alone for transrectal ultrasound-guided prostate biopsy without increased morbidities. Among the various local analgesics, lidocaine-prilocaine cream seems to offer the best overall efficacy.

Similarly Hiros M et al [17] studied in 90 patients who fulfilled the inclusion criteria were randomized into 3 groups of 30 patients each. Group 1 received periprostatic local anesthesia with $2 \%$ lidocaine, group 2 received Voltaren supp placed in rectum an hour before biopsy while group 3 received no local anesthesia. Pain scale responses were analyzed for each aspect of the biopsy procedure with a visual analog scale of 0 -none to 10-maximal. There was no difference between the 3 groups in pain scores during digital rectal examination, intrarectal injection and probe insertion. The mean pain scores during needle insertion in group 1 receiving periprostatic nerve block and in group 2 receiving Voltaren supp were 3,10 +/- 2,32 and 5,15 +/-
2,01 respectively. In group 3 (no local anesthesia), mean pain scores were $6,06+/-2,95$ which was found to be significantly different $(\mathrm{p}<0,001)$. However, morbidity after the biopsy was not statistically different between all 3 groups. TRUS-guided prostate biopsy is a traumatic and painful experience, but the periprostatic blockage use is clearly associated with more tolerance and patient comfort during the exam. It is an easy, safe, acceptable and reproducible technique and should be considered for all patients undergoing TRUS biopsy regardless of age or number of biopsies.

Peyromaure et al [18] found that only $47.6 \%$ of 275 patients described the procedure as painful (mild in two-thirds) on a visual analog scale (VAS), given adequate information before the procedure. By prospectively comparing a 12 with a 6 core biopsy protocol Naughton et al[19] noted no statistically significant difference in the 2 procedures in regard to any mean pain level at biopsy or subsequently. Zisman at al [20] evaluated the impact of prostate biopsy on patient well-being in 211 consecutive men in whom a mean of 8 biopsy cores was obtained. Immediate pain or discomfort was experienced during the procedure by $96 \%$ and $89 \%$ of their patients, respectively.

Another issue is the concept of pain accumulation, as introduced by Saha PK et al [21] They found that pain during biopsy gradually accumulates from the first core to the last one even when anesthesia is administered. Their study remains the only report to address the pain score at biopsy after each single sample.

Therefore it is concluded that peri-prostatic infiltration of $1 \%$ lignocaine provides pain relief during the TRUS guided prostate biopsy and thereby may decrease the early complications of the procedure.

\section{Conclusion}

Many patients have pain during transrectal ultrasound guided biopsies of the prostate and few clinicians provide a periprostatic nerve block before this procedure. A periprostatic nerve block administered before the biopsies dramatically decreases discomfort.

We urge all urologists to attempt this procedure, and we are confident that they will adopt it as part of their practice. 
The main limitation of the current study being small sample size. This is very simple technique and the skill can be acquired easily. We recommend nerve block during every TRUS guided prostate biopsy. However a future studies with a large number of patients should be carried out before adopting this recommendation.

\section{Funding: Nil, Conflict of interest: None Permission of IRB: Yes}

\section{References}

1. Parkin DM, Bray F, Ferlay J, Pisani P. Global cancer statistics, 2002. CA Cancer J Clin. 2005 Mar-Apr;55 (2): 74-108.

2. Nazir B Pain during transrectal ultrasound-guided prostate biopsy and the role of periprostatic nerve block: what radiologists should know Korean J Radiol. 2014; 15:543-553. www.ncbi.nlm.nih.gov/

3. Maccagnano C, Scattoni V, Roscigno M, Raber M, Angiolilli D, Montorsi F, Rigatti P. Anaesthesia in transrectal prostate biopsy: which is the most effective technique? Urol Int. 2011;87(1):1-13. doi: 10.1159/ 000327827. Epub 2011 Jun 16.

4. El-Hakim A, Moussa S. CUA guidelines on prostate biopsy methodology.Can Urol Assoc J. 2010 Apr;4(2): 89-94.

5. Soloway MS, Obek C. Periprostatic local anesthesia before ultrasound guided prostate biopsy. J Urol. 2000 Jan; 163(1):172-3.

6. Alavi AS, Soloway MS, Vaidya A, Lynne CM, Gheiler EL: Local anesthesia for ultrasound guided prostate biopsy: a prospective randomized trial comparing 2 methods. J Urol 2001;166:1343-1345.

7. Nash PA, Bruce JE, Indudhara R, Shinohara K. Transrectal ultrasound guided prostatic nerve blockade eases systematic needle biopsy of the prostate. J Urol. 1996 Feb;155(2):607-9.

8. Stirling BN, Shockley KF, Carothers GG, Maatman TJ: Comparison of local anesthesia techniques during transrectal ultrasound-guided biopsies. Urology 2002; 60:89-92.

9. Shinohara K, Master VA, Chi T, et al: Prostate needle biopsy techniques and interpretation; in
Vogelzang NJ, Scardino PT, Shipley WU (eds): Genitourinary Oncology. Philadelphia, Lippincott, Williams \& Wilkins, 2006, pp 111-119.

10. Babar Nazir Pain during Transrectal UltrasoundGuided Prostate Biopsy and the Role of Periprostatic Nerve Block: What Radiologists Should Know. Korean J Radiol. 2014 Sep-Oct; 15(5): 543-553.

11. Ramey JR, Halpern EJ, Gomella LG. Ultrasonography and Biopsy of the prostate. In: Wein AJ, Kavoussi LR, Novick AC, Partin AW, Peters CA, editors. Campbell-Walsh Urology. 9th ed. Philadelphia: Saunders; 2007. pp. 2883-2895.

12. Bulbul, M. A., Haddad, M. C., Khauli, R. B., Hemady, K., Shaar, A., Khouzami, R. et al: Periprostatic infiltration with local anaesthesia during transrectal ultrasound-guided prostate biopsy is safe, simple, and effective: a pilot study. Clin Imaging 2002; 26: 129-32.

13. Sabbagh R, McCormack M, Péloquin F, Faucher R, Perreault JP, Perrotte P, Karakiewicz PI, Saad F. A prospective randomized trial of 1-day versus 3-day antibiotic prophylaxis for transrectal ultrasound guided prostate biopsy. Can J Urol. 2004 Apr;11(2):2216-9.

14. Desgrandchamps F, Meria P, Irani J, Desgrippes A, Teillac P, Le Duc A. The rectal administration of lidocaine gel and tolerance of transrectal ultrasonography-guided biopsy of the prostate: a prospective randomized placebo-controlled study. BJU Int. 1999; 83:1007-9.

15. Wang $\mathrm{J}^{1}$, Wang $\mathrm{L}$, Addition of intrarectal local analgesia to periprostatic nerve block improves pain control for transrectal ultrasonography-guided prostate biopsy: a systematic review and meta-analysis. Int $\mathrm{J}$ Urol. 2015 Jan;22(1):62-8.

16. Woo HH Editorial Comment from Dr Woo to Addition of intrarectal local analgesia to periprostatic nerve block improves pain control for transrectal ultrasonography-guided prostate biopsy: a systematic review and meta-analysis. Int J Urol. 2015 Jan; 22 (1):68-9.

17. Hiros M, Selimovic M, Spahovic H, Sadovic S, Spuzic-Celic E. Transrectal ultrasound-guided prostate 


\section{Original Research Article}

biopsy, periprostatic local anesthesia and pain tolerance. Bosn J Basic Med Sci. 2010 Feb;10(1):68-72.

18. Peyromaure M, Ravery V, Messas A, Toublanc M, Boccon-Gibod L, Boccon-Gibod L. Pain and morbidity of an extensive prostate 10-biopsy protocol: a prospective study in 289 patients. J Urol. 2002 Jan;167(1):218-21.

19. Naughton CK, Ornstein DK, Smith DS, Catalona WJ. Pain and morbidity of transrectal ultrasound guided prostate biopsy: a prospective randomized trial of 6 versus 12 cores. J Urol. 2000 Jan;163(1):168-71.

20. Zisman A, Leibovici D, Kleinmann J, Siegel YI, Lindner A. The impact of prostate biopsy on patient well-being: a prospective study of pain, anxiety and erectile dysfunction. J Urol. 2001 Feb;165(2):445-54.

21.Saha PK, Alam SM, Khatun US, Rahman MH, Gupta SD. Use of local anesthesia in transrectal ultrasound guided prostatic biopsy. Mymensingh Med J. 2014 Apr;23(2):299-304.

\section{How to cite this article?}

Ali Q, Grower J. A study of the pain score following TRUS guided prostate biopsy with and without periprostatic lignocaine infiltration. Int J Med Res Rev 2017;5(04):393-398 doi:10.17511/ijmrr. 2017.i04.04. 